\title{
LA DIVERSIFICACIÓN GEOGRÁFICA INTERNACIONAL DE LAS CADENAS HOTELERAS ESPAÑOLAS EN AÑOS DE CRISIS
}

\author{
Rosario Andreu \\ Enrique Claver \\ Diego Quer \\ Universidad de Alicante
}

\section{RESUMEN}

Dentro del sector turístico, el sector hotelero español representa uno de los sectores más dinámicos. Prueba de ello es la gran cantidad de decisiones estratégicas de crecimiento que son tomadas continuamente por las empresas hoteleras españolas. Entre estas estrategias de crecimiento, el presente trabajo se centra en las estrategias de diversificación geográfica. Concretamente, se analiza la evolución en la diversificación internacional de las cadenas hoteleras españolas durante los años 2008-2014 y su relación con la rentabilidad empresarial. Para complementar el análisis, también se analiza la relación entre la diversificación y otras variables empresariales como el tamaño y la antigüedad.

Palabras clave: cadenas hoteleras, diversificación geográfica, rentabilidad, tamaño, antigüedad.

International geographic diversification of Spanish hotel chains during the crisis period

\section{ABSTRACT}

The hotel sector is one of the most dynamic tourism industries in Spain and one of the most important industries for the Spanish economy. Proof of this is the large number of growth strategies that are continuously carried out by Spanish hotel companies. Within these growth stra-

Recibido: 21 de diciembre de 2015

Devuelto para su revisión: 4 de mayo de 2016

Aceptado: 25 de julio de 2016

Departamento de Organización de Empresas. Facultad de Ciencias Económicas y Empresariales. Universidad de Alicante. Campus de San Vicente del Raspeig s/n. 03690 ALICANTE (España).E-mail: rosario.andreu@ua.es, enrique.claver@ua.es,diego.quer@ua.es 
tegies, this paper focuses on geographic diversification strategies. More precisely, we analyze the evolution of the international diversification of Spanish hotel chains during the years 20082014 and its relationship with performance. Moreover, we investigate the relationship between geographic diversification and other firm-specific factors such as size and age.

Keywords: hotel chains, geographical diversification, performance, size, age.

\section{INTRODUCCIÓN}

Uno de los motores de la economía española durante los últimos años ha sido el turismo. Según los cálculos realizados a partir del último dato oficial publicado por el INE (2013), el turismo supone más el 11\% del PIB español. Incluso se estima que el peso de la actividad turística pueda llegar a alcanzar el 15\% en 2022 (Figuerola, 2014). Los datos demuestran que el turismo ha sido uno de los sectores que menos ha notado la crisis, actuando como un "estabilizador de la economía española" (García, 2014: 25). Según las estadísticas de EXCELTUR (2015), el PIB turístico ha crecido en los últimos años por encima del PIB español. Las perspectivas para 2015 situaban el crecimiento del PIB turístico en un $3,6 \%$, lo que supone medio punto más que el crecimiento del conjunto de la economía española. España sigue, además, ocupando las primeras posiciones de los rankings mundiales de turismo al ser el tercer país en cuanto a llegadas de turistas internacionales y el segundo en cuanto a ingresos por turismo (UNWTO, 2015).

Dentro del sector turístico, el sector hotelero español ha sabido hacerse un hueco indiscutible a nivel mundial. Las principales cadenas hoteleras españolas ocupan, año tras año, puestos de relevancia en los rankings mundiales (Hotels, 2015). A pesar de la crisis, desde comienzos de la década actual el sector hotelero español ha venido experimentando un crecimiento, tanto en número de hoteles como en número de camas, que alcanzó su tasa más alta en 2011 (INE, 2015). En las últimas décadas, además, la industria hotelera española ha experimentado un crecimiento espectacular en su grado de internacionalización (Brida, Driha, Ramón-Rodríguez y Scuderi, 2015). Como señalan Martorell y Mulet (2013), la capacidad de alojamiento que presenta el sector hotelero español permite que goce de un gran reconocimiento en el panorama internacional.

La importancia alcanzada por el sector hotelero ha llevado a que sea uno de los más estudiados dentro de la investigación científica sobre turismo (Corral, 2014; López, 2010). Son muchos los trabajos centrados en analizar diferentes aspectos de las cadenas hoteleras españolas desde distintas perspectivas. Temas como la gestión de la calidad o medioambiental de los hoteles, la responsabilidad social corporativa, la innovación, o la gestión de recursos humanos, entre otros, han sido algunos de los más abordados por la literatura académica.

Dentro de estas líneas de investigación, cabe destacar la que se centra en el análisis de la estrategia empresarial de crecimiento y, más concretamente, en las decisiones sobre internacionalización y diversificación de las cadenas hoteleras españolas, estudiadas en trabajos previos como los de Berbel y Ramírez (2005), Claver, Andreu y Quer (2006 y 2007), Claver, Quer y Andreu (2006), Driha y Ramón (2011), Martorell, Mulet y Otero 
(2013), Pérez y Cazorla (2002) o Pla-Barber, León-Darder y Villar (2011), entre otros. Nuestro trabajo se encuadra en esta línea al analizar la tendencia general seguida por las cadenas hoteleras españolas más importantes en cuanto a su estrategia de diversificación de los mercados geográficos. A pesar de la importancia de las estrategias de internacionalización para la industria hotelera española, existen pocos trabajos que hayan analizado su evolución, como apuntan Brida, Ramón-Rodríguez, Such-Devesa y Driha (2016). De acuerdo con estos autores, se pueden identificar diferentes etapas en el proceso de internacionalización de las empresas hoteleras españolas, cuyo comienzo se sitúa en los años 80. En los años 90, la internacionalización se acelera como una forma de diversificar el riesgo y dar respuesta a la crisis turística. Las empresas hoteleras se establecieron principalmente en América Latina. Son empresas especializadas en turismo vacacional. A partir del año 2000, la madurez alcanzada por el turismo vacacional lleva a que las empresas decidan diversificar hacia el turismo urbano y de negocios estableciéndose en mayor medida en destinos europeos. En los últimos años, la globalización ha facilitado la incorporación de pequeñas y medianas empresas como nuevos competidores en los mercados internacionales, a pesar de que sigue existiendo una gran concentración de la oferta hotelera en el exterior en manos de las principales cadenas hoteleras españolas. Por otra parte, también han surgido nuevos destinos turísticos emergentes por los que están apostando en los últimos años algunas de las principales cadenas hoteleras españolas. Todos estos hechos, junto con la crisis económica mundial de los últimos años, han provocado cambios en la estructura del sector a nivel internacional.

El objetivo de nuestro trabajo será determinar el nivel de internacionalización y de diversificación geográfica que las empresas hoteleras españolas han alcanzado durante los últimos años, analizando además su relación con la rentabilidad empresarial. Describiremos la evolución de la internacionalización de las cadenas hoteleras españolas durante los años de crisis, situando el horizonte temporal a comienzos de la misma, en 2008. También se analizará la relación de la diversificación geográfica internacional con otra serie de características de las empresas objeto de estudio como su tamaño o su antigüedad, tratando así de obtener información que pueda resultar de interés para futuras investigaciones.

Para cumplir nuestro propósito, en primer lugar realizaremos una revisión teórica sobre algunos de los aspectos más importantes que han sido abordados sobre las estrategias de diversificación empresarial. A continuación, mostraremos el trabajo empírico realizado y los resultados obtenidos. Finalizaremos destacando las principales aportaciones de nuestro trabajo a modo de conclusión.

\section{ESTRATEGIAS DE DIVERSIFICACIÓN EMPRESARIAL}

Las estrategias de diversificación son consideradas desde diferentes teorías como una de las estrategias más importantes para mejorar los resultados de las empresas. Por este motivo, han sido muchos los estudios que se han realizado para analizar la influencia de esta estrategia sobre la rentabilidad empresarial. Entre ellos, podemos citar los trabajos de Berger y Ofeck (1995), Bettis (1981), Chen y Chang (2012), Christensen, y Montgomery (1981), Lee y Jang (2007), Montgomery y Wernerfelt (1988), Pandya y Rao (1998), Ramírez y Espitia (2002), Rumelt (1974 y 1982) o Sing y Gu (1994). 
Sin embargo, las diferencias en las medidas usadas y en el diseño de los análisis empíricos de estos trabajos, han llevado a que los resultados alcanzados sean muy diversos y, por tanto, no concluyentes (Huerta y Navas, 2007; Benito, 2009). De esta forma, no se puede asegurar la superioridad de ningún tipo de estrategia de diversificación, aunque la idea más generalizada tiende a apoyar la tesis original de Rumelt (1974) sobre la existencia de una relación positiva entre la diversificación relacionada y los resultados empresariales y una relación negativa en el caso de la diversificación no relacionada.

Las diferentes formas de medir los niveles o los tipos de diversificación en la empresa han sido otro de los temas más abordados, junto con los motivos que llevan a la empresa a diversificar su actividad. En este sentido, Hoskisson y Hitt (1990) realizaron una síntesis de los motivos que justifican dicha estrategia y que pueden ser agrupados en motivos financieros y motivos de búsqueda de sinergias. El principal motivo financiero para la diversificación es la reducción del riesgo de la empresa ya que puede disminuir la variabilidad del flujo de beneficios, aunque el tipo de diversificación que permite una mayor reducción del riesgo tampoco está clara. Trabajos como el de Amit y Livnat (1988) encontraron que la diversificación no relacionada va asociada a los menores niveles de riesgo, mientras que Lubatkin y Chatterjee (1994) llegaron a la conclusión de que los menores niveles de riesgo se detectan cuando la empresa presenta una diversificación más relacionada. Por su parte, la posibilidad de explotar sinergias es la principal justificación desde la Dirección Estratégica (Markides y Williamson, 1994) ya que ayuda a la empresa a aumentar su tamaño, a lograr economías de escala o de alcance y a aumentar su poder de mercado (Lee y Jang, 2007).

La mayoría de los estudios anteriormente citados han abordado el tema de la diversificación empresarial centrándose en la diversificación de producto o de negocio. Sin embargo, como señalan Jiménez-Palmero y Benito-Osorio (2011), el concepto de diversificación puede estudiarse desde una doble perspectiva: la basada en el producto y la geográfica. Nuestro trabajo se centra en la dimensión geográfica de la diversificación, siguiendo así la línea de trabajos como los de Capar y Kotabe (2003) o Lu y Beamish (2004).

La diversificación geográfica ha sido objeto de estudio en numerosas investigaciones en los últimos años. Muchas de estas investigaciones se han centrado en analizar la influencia de la diversificación internacional en los resultados empresariales Sin embargo, al igual que sucedía con el caso de la diversificación de producto, tampoco existe consenso sobre si las empresas con un mayor nivel de diversificación internacional presentan unos mayores beneficios.

Por una parte, existen estudios que han obtenido una relación lineal y positiva entre la dispersión geográfica y la rentabilidad (Delios y Beamish, 1999; Kim, Hwang y Burgers, 1993), una relación negativa (Colpan, 2008; Geringer, Tallman y Olsen, 2000), o ninguna relación (Hennart, 2007; Morck y Yeung, 1991). Por otra parte, existen trabajos que han obtenido una relación curvilínea, tanto en forma de U (Capar y Kotabe, 2003; Lu y Beamish, 2001), como en forma de U invertida (Geringer, Beamish y daCosta, 1989; Hit, Hoskisson y Kim, 1997). Por último, también se ha planteado la posibilidad de que exista una relación en forma de $\mathrm{S}$ horizontal entre el grado de diversificación internacional y la rentabilidad empresarial, habiéndose confirmado en algunos trabajos (Lu y Beamish, 2004) pero no en otros (Jiménez-Palmero y Benito-Osorio, 2011). 
De nuevo, las diferencias en la forma en que es medida la diversificación geográfica puede ser una de las causas de los resultados tan dispares. Existen trabajos que han usado como medida de diversificación internacional el porcentaje que suponen las ventas internacionales sobre las ventas totales de la empresa (Gomes y Ramaswamy, 1999) o la inversión en el exterior sobre el total de activos (Capar y Kotabe, 2003). Por otra parte, trabajos como los de Jiménez-Palmero y Benito-Osorio (2011), Lu y Beamish (2004) o Tallman y Li (1996), han usado el número de países donde opera la empresa como aproximación de la dispersión geográfica. Por último, también existen trabajos que han utilizado medidas categóricas para clasificar a las empresas en función de su mayor o menor amplitud geográfica, basándose para ello en las categorías propuestas por Varadarajan (1986) y Varadarajan y Ramanujam (1987). Es el caso de los trabajos de Kranenburg, Hagendoorn y Pennings (2003) o Ramírez y Espitia (2000).

A pesar de la gran cantidad de trabajos que existen centrados en esta estrategia de crecimiento diversificado, en el sector hotelero la literatura sobre diversificación empresarial no es muy abundante. Pocos trabajos han analizado la tendencia reciente en la diversificación de este sector y las implicaciones sobre la rentabilidad de las empresas. Una excepción la encontramos en los trabajos de Chen y Chang (2012) y Lee y Jang (2007), quienes analizaron la relación entre diferentes tipos de diversificación, con el riesgo y los resultados empresariales (tanto económicos como financieros) para el caso de empresas hoteleras de Taiwán y Estados Unidos, respectivamente. Las conclusiones de estos trabajos apuntan a que la rentabilidad de las empresas hoteleras viene condicionada por la diversificación. En ambos trabajos se obtiene que las empresas hoteleras más diversificadas son las que presentan una mayor estabilidad en los resultados y, por tanto, un menor riesgo.

Por otra parte, la mayoría de los trabajos que han analizado la diversificación geográfica o internacional y su relación con la rentabilidad empresarial, han incluido a empresas de diferentes sectores, sin que existan apenas trabajos específicos centrados en empresas del sector servicios en general y del sector hotelero en particular. Por este motivo, el presente trabajo indaga en este gap detectado, analizando la diversificación geográfica internacional del sector hotelero español en los últimos años y su relación con los resultados empresariales. A partir de este análisis, se pretende obtener un punto de partida para realizar futuras investigaciones.

\section{METODOLOGíA}

Para alcanzar los objetivos de nuestro trabajo, hemos empleado fuentes de datos secundarias con el fin de recopilar la información necesaria para realizar el estudio de las empresas que forman parte de la muestra. A continuación, describimos el estudio empírico planteado comenzando por la muestra y las variables definidas.

\subsection{Muestra y período analizado}

Nuestra muestra la integran las cadenas hoteleras españolas con más presencia internacional en el año 2014, de acuerdo con el ranking de presencia internacional publicado anualmente por la revista Hosteltur. En total, son 61 las empresas que formaban parte de 
este ranking en el año 2014. Se ha estudiado la evolución en la diversificación geográfica de estas empresas durante el período 2008-2014. Para conocer dicha evolución se han consultado los rankings de Hosteltur de estos años. Además, para contrastar y completar la información de las empresas de la muestra se han consultado los anuarios de Hostelmarket (2015) y la base de datos SABI (Sistema de Análisis de Balances Ibéricos).

\subsection{Variables}

\subsubsection{Diversificación geográfica}

Para medir la diversificación geográfica de las cadenas hoteleras españolas se han empleado dos variables, una continua y otra categórica, tomando como referencia los diferentes trabajos que han sido comentados en un apartado anterior.

En primer lugar, se ha utilizado el número total de países donde las empresas tienen presencia internacional ${ }^{1}$. Esta medida ha sido utilizada en los trabajos de Jiménez-Palmero y Benito-Osorio (2011), Lu y Beamish (2004) y Tallman y Li (1996). En segundo lugar, también se ha optado por medir el grado de diversificación internacional de manera categórica, siguiendo la propuesta de Ramírez y Espitia (2000). De esta forma, se han clasificado las empresas en función del "espectro amplio de diversificación internacional (BSDI)" y el "espectro medio de diversificación internacional (MNSDI)". El BSDI se refiere a la expansión en diferentes áreas geográficas ${ }^{2}$ mientras que el MNSDI es el número medio de países en los que está presente la empresa por área geográfica. Estos espectros se dividen en dos categorías: alto y bajo, cuyo punto de corte lo determinan los valores medios de la muestra. Combinando ambas dimensiones se obtienen las cuatro categorías de diversificación internacional que se muestran en la siguiente tabla.

\section{Tabla 1 \\ TIPOLOGÍA DE ESTRATEGIAS DE DIVERSIFICACIÓN INTERNACIONAL}

\begin{tabular}{|c|c|c|c|}
\hline \multirow{3}{*}{ BSDI } & Bajo & $\begin{array}{c}\text { Empresas con muy baja diversidad } \\
\text { internacional (BDI) }\end{array}$ & $\begin{array}{c}\text { Empresas diversificadas relacionadas } \\
\text { internacionalmente (DRI) }\end{array}$ \\
\hline & Alto & $\begin{array}{c}\text { Empresas diversificadas no } \\
\text { relacionadas internacionalmente } \\
\text { (DNRI) }\end{array}$ & $\begin{array}{l}\text { Empresas con muy alta diversidad } \\
\text { internacional (ADI) }\end{array}$ \\
\hline & & Bajo & Alto \\
\hline
\end{tabular}

Fuente: Ramírez y Espitia (2000).

1 Otra variable definida para medir la diversificación geográfica fue el porcentaje del número de habitaciones en el exterior sobre el total de habitaciones de la empresa. Sin embargo, esta variable resultó estar altamente correlacionada con la variable número de países, por lo que se optó por esta última al ser la más comúnmente empleada en trabajos previos.

2 Las áreas geográficas consideradas en este estudio han sido cuatro: Europa y Cuenca Mediterránea, Caribe, resto de América y África y Asia. 
Siguiendo a Ramírez y Espitia (2000), hemos reagrupado estas cuatro estrategias en dos. Las empresas incluidas en las categorías DNRI y ADI quedarían clasificadas en la categoría de Gran Diversidad Internacional (GDI), mientras que las incluidas en las categorías BDI y DRI quedarían clasificadas como Poca Diversidad Internacional (PDI). De esta forma, hemos definido una variable categórica con dos posibles valores: GDI y PDI.

\subsubsection{Rentabilidad Empresarial}

Para medir los resultados de la empresa se ha utilizado el ROA (Resultados sobre los Activos). El ROA es calculado dividiendo la rentabilidad antes de impuestos entre el total de activos de la empresa. Esta medida de la rentabilidad ha sido comúnmente empleada en los trabajos realizados sobre diversificación, tanto de producto como geográfica. Entre ellos, podemos mencionar el de Berger y Ofek (1995), Bettis, (1981), Davis, Robinson, Pearce y Park (1992), Dubofsky y Varadarajan (1987), Grant, Jamine y Thomas (1988), Hall y John (1994), Hitt, Hoskisson y Kim (1997), Li y Greenwood (2004), Lu y Beamish, (2004) o Park (2002 y 2003).

\subsubsection{Tamaño de la empresa}

En cuanto al tamaño empresarial, ha sido aproximado mediante el número de habitaciones de la empresa en el año 2014. Esta variable ha sido utilizada en trabajos previos centrados en el sector hotelero como el de Camisón (2000). Dado que el rango de esta variable era muy grande, se ha realizado una transformación logarítmica.

\subsubsection{Antigüedad de la empresa}

A partir del año de creación de la empresa se ha obtenido la antigüedad que presenta la empresa en el año 2014. Esta variable ha sido utilizada en el análisis de la diversificación geográfica de las multinacionales españolas por Jiménez-Palmero y Beniro-Osorio (2011).

\subsubsection{Principal zona geográfica en su internacionalización}

Para calcular cuál es la principal zona en la internacionalización de cada empresa, en primer lugar se ha procedido a calcular el número de habitaciones que la empresa posee en cada una de las áreas geográficas consideradas. A continuación, se ha calculado el porcentaje que suponen las habitaciones de cada área en el total de habitaciones en el exterior de la empresa. Cada empresa ha sido clasificada en aquella zona geográfica donde posee el mayor porcentaje de habitaciones.

\section{RESULTADOS}

Para realizar nuestro estudio empírico hemos utilizado técnicas de análisis bivariables no paramétricas, realizadas con el programa SPSS versión 22. La prueba de KolmogorovSmirnoff, para comprobar si las variables siguen una distribución normal, nos lleva a 
rechazar la hipótesis de normalidad, por lo que debemos optar por técnicas de análisis no paramétricas. Concretamente, utilizaremos el coeficiente de correlación rho de Spearman, la prueba U de Mann-Whitney y la prueba de Kruskal-Wallis (Chi-cuadrado). Los resultados de dichas pruebas se presentan en el siguiente apartado.

\subsection{Análisis descriptivo}

En la siguiente tabla presentamos las principales características de las empresas que forman parte de la muestra en el año 2014.

Tabla 2

VALORES DESCRIPTIVOS DE LAS EMPRESAS AÑO 2014

\begin{tabular}{|c|c|c|}
\hline Tamaño medio & Antigüedad media & Rentabilidad Media \\
\hline 7.479 habitaciones & 21,48 años & $0,0033(\mathrm{en} \%)$ \\
\hline $\mathrm{N}=61$ & $\mathrm{~N}=61$ & $\mathrm{~N}=54$ \\
\hline
\end{tabular}

Podemos ver que el tamaño medio de las empresas de la muestra es alrededor de 7.500 habitaciones, la antigüedad supera los 21 años, mientras que la rentabilidad media se sitúa en un nivel muy bajo cercano al $0 \%$. En cuanto a la evolución en su grado de diversificación geográfica durante el período analizado, los datos se recogen en la siguiente tabla.

Tabla 3

EVOLUCIÓN DEL GRADO DE INTERNACIONALIZACIÓN DE LAS EMPRESAS DE LA MUESTRA

\begin{tabular}{|l|c|c|c|c|c|c|c|}
\hline \multicolumn{1}{|c|}{ Año } & $\mathbf{2 0 0 8}$ & $\mathbf{2 0 0 9}$ & $\mathbf{2 0 1 0}$ & $\mathbf{2 0 1 1}$ & $\mathbf{2 0 1 2}$ & $\mathbf{2 0 1 3}$ & $\mathbf{2 0 1 4}$ \\
\hline $\begin{array}{l}\text { Número total de } \\
\text { habitaciones en el exterior }\end{array}$ & 224.265 & 224.451 & 232.304 & 232.997 & 226.972 & 228.317 & 232.973 \\
\hline $\begin{array}{l}\text { \% habitaciones en el } \\
\text { exterior sobre el total de } \\
\text { habitaciones de la empresa }\end{array}$ & $39 \%$ & $36,63 \%$ & 35,87 & 35,95 & 35,45 & 35,13 & 35,98 \\
\hline $\begin{array}{l}\text { Número medio de países } \\
\text { distintos donde está } \\
\text { presente la empresa }\end{array}$ & 4,71 & 4,45 & 3,66 & 3,67 & 3,79 & 3,95 & 3,89 \\
\hline \multicolumn{1}{|c|}{$\mathbf{N}$} & $\mathbf{3 8}$ & $\mathbf{4 2}$ & $\mathbf{5 8}$ & $\mathbf{6 1}$ & $\mathbf{5 8}$ & $\mathbf{5 8}$ & $\mathbf{6 1}$ \\
\hline
\end{tabular}

Como podemos observar, desde el año 2008 el número de habitaciones en el exterior ha aumentado año tras año, exceptuando el año 2012 en que se produjo una fuerte caída de unas 6.000 habitaciones. No obstante, en 2013 se muestra una recuperación de las cifras, registrando en el año 2014 un aumento respecto al año 2013 de 4.000 habitaciones (aproximadamente un 2\% más). En cuanto al porcentaje que suponen estas habitaciones 
en el exterior sobre el total de habitaciones de la empresa, se ha producido una reducción si consideramos todo el período analizado, aunque se observa una tendencia al alza en el último año. En este último año, el porcentaje roza el 36\%. Es decir, de media, el 36\% de las habitaciones de las empresas de la muestra se encuentran en el exterior. A comienzos de la crisis, el porcentaje ascendía al 39\%. Por su parte, el número medio de países distintos donde las empresas están presentes ha disminuido durante el período analizado, situándose en cerca de los 4 países de media. Analizamos ahora en la siguiente tabla el reparto de habitaciones por área geográfica.

Tabla 4

REPARTO DE HABITACIONES POR ÁREA GEOGRÁFICA

\begin{tabular}{|c|c|c|c|c|}
\hline \multirow{2}{*}{ Año } & $\begin{array}{c}\text { Europa y } \\
\text { Cuenca Mediterránea }\end{array}$ & Caribe & $\begin{array}{c}\text { Resto de } \\
\text { América }\end{array}$ & África y Asia \\
\hline \multirow{2}{*}{2008} & 85.169 & 111.442 & 24.171 & 3.483 \\
& $(37,98 \%)$ & $(49,70 \%)$ & $(10,78 \%)$ & $(1,55 \%)$ \\
\hline \multirow{2}{*}{$\mathbf{2 0 0 9}$} & 85.978 & 110.117 & 23.814 & 4.542 \\
& $(38,30 \%)$ & $(49,06 \%)$ & $(10,61 \%)$ & $(2,02 \%)$ \\
\hline \multirow{2}{*}{$\mathbf{2 0 1 0}$} & 89.033 & 113.138 & 25.193 & 4.940 \\
& $(38,33 \%)$ & $(48,7 \%)$ & $(10,84 \%)$ & $(2,13 \%)$ \\
\hline \multirow{2}{*}{$\mathbf{2 0 1 1}$} & 89.842 & 115.660 & 21.550 & 5.945 \\
& $(38,56 \%)$ & $(49,64 \%)$ & $(9,25 \%)$ & $(2,6 \%)$ \\
\hline \multirow{2}{*}{$\mathbf{2 0 1 2}$} & 85.662 & 113.876 & 21.167 & 6.267 \\
& $(37,74 \%)$ & $(50,17 \%)$ & $(9,33 \%)$ & $(2,76 \%)$ \\
\hline \multirow{2}{*}{$\mathbf{2 0 1 3}$} & 86.277 & 115.476 & 20.251 & 6.317 \\
& $(37,79 \%)$ & $(50,58 \%)$ & $(8,87 \%)$ & $(2,77 \%)$ \\
\hline \multirow{2}{*}{$\mathbf{2 0 1 4}$} & 86.333 & 119.882 & 20.687 & 6.098 \\
& $(37 \%)$ & $(51,5 \%)$ & $(8,9 \%)$ & $(2,6 \%)$ \\
\hline
\end{tabular}

La tabla anterior muestra que la principal zona de internacionalización de las cadenas hoteleras españolas ha sido, en todos los años analizados, la del Caribe. Esta zona, además, ha experimentado el mayor crecimiento en el número de habitaciones, ascendiendo dicho aumento a más de 8.000 habitaciones en los últimos 7 años. Por su parte, las zonas de Europa-Mediterráneo y el resto de América han experimentado una disminución en el número de camas, tomando como referencia el dato de 2008, aunque se observa un ligero aumento en el año 2014 con respecto al año 2013. Cabe destacar también que la internacionalización hacia la zona de África y Asia ha sido la otra zona que ha crecido en el período analizado, doblando casi el número de habitaciones con respecto a 2008, si bien en este caso se muestra una tendencia decreciente desde el año 2012.

Por países, en la siguiente tabla se muestran los diez principales destinos de las cadenas hoteleras españolas en el año 2014. Como se observa, México, República Dominicana y Cuba encabezan este ranking. 
Tabla 5

TOP TEN DESTINOS INTERNACIONALES

DE LAS CADENAS HOTELERAS ESPAÑOLAS EN 2014

\begin{tabular}{|l|c|}
\hline \multicolumn{1}{|c|}{ País } & Número de habitaciones \\
\hline 1. México & 41.241 \\
\hline 2. República Dominicana & 37.434 \\
\hline 3. Cuba & 25.569 \\
\hline 4. Alemania & 17.781 \\
\hline 5. Italia & 13.787 \\
\hline 6. EE.UU. & 8.399 \\
\hline 7. Túnez & 7.249 \\
\hline 8. Holanda & 7.099 \\
\hline 9. Croacia & 6.908 \\
\hline 10. Brasil & 5.837 \\
\hline
\end{tabular}

Pasaremos a continuación a mostrar las pruebas estadísticas realizadas para comprobar la relación entre la diversificación geográfica y la rentabilidad.

\subsection{Relación entre diversificación geográfica y rentabilidad empresarial}

Para analizar si existe correlación entre el nivel de diversificación geográfica que presentan las empresas de la muestra en el año 2014 y la rentabilidad económica de ese año utilizamos el coeficiente Rho de Spearman. Dado que para 7 de las empresas de la muestra no se dispone de los datos de rentabilidad relativos a 2014, el número total de casos para esta prueba se ha reducido a 54.

\section{Tabla 6 \\ CORRELACIÓN ENTRE EL GRADO DE DIVERSIFICACIÓN GEOGRÁFICA Y LA RENTABILIDAD}

\begin{tabular}{|l|c|c|}
\hline \multicolumn{2}{|c|}{} & Número de países \\
\hline \multirow{3}{*}{ Rentabilidad } & Coeficiente Rho de Spearman & $\begin{array}{c}-0,029 \\
(\text { Sig. 0,836) }\end{array}$ \\
\cline { 2 - 3 } & N & 54 \\
\hline
\end{tabular}

Como se observa en la tabla anterior, la relación resultante no es significativa, por lo que no se puede afirmar que la diversificación geográfica y la rentabilidad empresarial estén relacionadas. Además, el coeficiente muestra un signo negativo, aunque éste sea muy bajo. Esto podría indicar que la diversificación geográfica va asociada a una menor renta- 
bilidad, pero no quiere decir que la relación sea de causa y efecto. Es decir, esta relación no nos permitiría saber si la causa de la menor rentabilidad es la diversificación geográfica o si es que son las empresas con una menor rentabilidad las que más diversifican.

En la siguiente tabla se muestra la relación con la otra variable categórica empleada para medir la diversificación geográfica, empleando para ello la prueba U de MannWhitney.

Tabla 7

PRUEBA U DE MANN-WHITNEY PARA GRADO DE DIVERSIFICACIÓN GEOGRÁFICA PDI-GDI Y RENTABILIDAD

\begin{tabular}{|c|c|c|c|c|}
\hline & Nivel de diversificación & N & $\begin{array}{c}\text { Rango } \\
\text { Promedio }\end{array}$ & Estadístico \\
\hline \multirow{3}{*}{ Rentabilidad } & PDI & 36 & 29,10 & \multirow{2}{*}{\begin{tabular}{c}
266,500 \\
\cline { 2 - 4 }
\end{tabular}} \\
\cline { 2 - 4 } & GDI & 18 & 24,31 & (Sig. 0,291) \\
\hline
\end{tabular}

De nuevo, la relación no es significativa. Se observa, además, que las empresas con Gran Diversificación Internacional muestran un menor promedio de rentabilidad, en la misma línea que lo señalado en la prueba anterior para la otra variable. Esto podría deberse a los costes asociados al proceso de internacionalización, que pueden ser más altos para las empresas que presentan una mayor presencia internacional, disminuyendo así sus beneficios. Estos resultados también podrían venir condicionados por la medida de rentabilidad empleada o por la situación económica tan complicada de las empresas en los últimos años, que puede haber distorsionado las cifras de la rentabilidad. En cualquier caso, no podemos confirmar que exista una relación entre la diversificación internacional y la rentabilidad, en la línea de lo obtenido en trabajos previos como los de Hennart (2007) o Jiménez-Palmero y Benito-Osorio (2011).

A continuación analizaremos si existen diferencias en la rentabilidad entre las principales zonas de internacionalización de las empresas de la muestra. Para realizar este contraste utilizaremos la prueba de Kruskal-Wallis, recogida en la siguiente tabla.

\section{Tabla 8}

PRUEBA KRUSKAL-WALLIS PARA LA ZONA PRINCIPAL DE INTERNACIONALIZACIÓN Y RENTABILIDAD

\begin{tabular}{|l|l|c|c|c|}
\hline & Zona Geográfica Principal & N & $\begin{array}{c}\text { Rango } \\
\text { Promedio }\end{array}$ & Chi-cuadrado \\
\hline \multirow{4}{*}{ Rentabilidad } & Europa y Cuenca Mediterránea & 27 & 26,39 & \\
\cline { 2 - 4 } & Caribe & 21 & 31,93 & \multirow{4}{4}{$\begin{array}{c}4,472 \\
\text { (Sig. 0,098) }\end{array}$} \\
\cline { 2 - 4 } & Resto de América & 6 & 17,00 & \\
\cline { 2 - 4 } & Total & 54 & & \\
\hline
\end{tabular}


Los resultados muestran que existen diferencias en la rentabilidad en función de cuál sea la principal zona de internacionalización de la empresa, con un nivel de significación del $10 \%$. La mayor rentabilidad la muestran las empresas cuya principal zona de internacionalización es el Caribe, seguida de la zona de Europa-Cuenca Mediterránea y, por último, Resto de América. Respecto a la zona de África y Asia, no existe ninguna empresa que la tenga como principal zona de internacionalización. Sin embargo, esta prueba no nos proporciona suficiente información para poder concluir que una zona geográfica presenta una mayor rentabilidad frente al resto. Para poder confirmar estadísticamente dicha relación debemos realizar un análisis por pares mediante la prueba U de Mann-Whitney, que es la equivalente no paramétrica a la prueba T de Student. Dado que no hay ninguna empresa cuya principal zona sea África y Asia, compararemos Europa-Caribe, EuropaResto de América y Caribe-Resto de América.

\section{Tabla 9}

PRUEBA U DE MANN-WHITNEY PARA LA ZONA PRINCIPAL DE INTERNACIONALIZACIÓN Y RENTABILIDAD

\begin{tabular}{|c|c|c|c|c|}
\hline & Zona Principal & $\mathbf{N}$ & $\begin{array}{c}\text { Rango } \\
\text { promedio }\end{array}$ & Estadístico \\
\hline \multirow{3}{*}{ Rentabilidad } & Europa-Mediterráneo & 27 & 22,24 & \multirow{3}{*}{$\begin{array}{c}222,5 \\
\text { (Sig. 0,205) }\end{array}$} \\
\hline & Caribe & 21 & 27,40 & \\
\hline & Total & 48 & & \\
\hline \multirow{3}{*}{ Rentabilidad } & Europa-Mediterráneo & 27 & 18,15 & \multirow{3}{*}{$\begin{array}{c}50,00 \\
\text { (Sig. 0,148) }\end{array}$} \\
\hline & Resto América & 6 & 11,83 & \\
\hline & Total & 33 & & \\
\hline \multirow{3}{*}{ Rentabilidad } & Caribe & 21 & 15,52 & \multirow{3}{*}{$\begin{array}{c}31,00 \\
\text { (Sig. 0,062) }\end{array}$} \\
\hline & Resto América & 6 & 8,67 & \\
\hline & Total & 27 & & \\
\hline
\end{tabular}

De los resultados se concluye que no se puede afirmar que la rentabilidad de las empresas que se han internacionalizado principalmente en el Caribe sea superior que las que lo han hecho en Europa dado que la relación no ha resultado ser estadísticamente significativa. Tampoco se puede confirmar que las que tienen presencia mayoritariamente en Europa se asocien con una mayor rentabilidad que las del Resto de América por el mismo motivo, el p-valor supera el 0,1. La única diferencia significativa, de nuevo al 10\%, se da entre las empresas de la zona Caribe y el Resto de América. Sólo en este último caso podemos concluir que existen diferencias estadísticamente significativas en la rentabilidad media, siendo más alta la de las empresas que tienen la zona del Caribe como principal zona de expansión internacional frente a las del Resto de América.

Por último, con el fin de complementar nuestro análisis y obtener información adicional sobre la diversificación geográfica, analizamos también la relación con otras variables de la empresa como el tamaño y la antigüedad. 


\subsection{Relación entre diversificación geográfica, tamaño y antigüedad de la empresa}

En la siguiente tabla se muestra la correlación entre las variables.

\section{CORRELACIÓN ENTRE EL TAMAÑO, ANTIGÜEDAD Y EL GRADO DE DIVERSIFICACIÓN GEOGRÁFICA}

\begin{tabular}{|l|c|c|}
\hline \multicolumn{2}{|c|}{ Tamaño } & Número de países \\
\hline Antigüedad & $\begin{array}{c}\text { Coeficiente } \\
\text { Rho de Spearman }\end{array}$ & $\begin{array}{c}0,631 \\
\text { (sig. 0,000) }\end{array}$ \\
\cline { 3 - 3 } & & $\begin{array}{c}0,251 \\
\text { (sig. 0,051) }\end{array}$ \\
\hline & $\mathbf{N}$ & 61 \\
\hline
\end{tabular}

Como se puede observar, el tamaño y la antigüedad están relacionados con el grado de diversificación geográfica de manera positiva y significativa, al $1 \%$ y al $10 \%$ respectivamente, cuando utilizamos como medida el número de países en los que está presente la empresa. Por tanto, las empresas de mayor tamaño y de mayor antigüedad son también las más diversificadas internacionalmente. En el caso del tamaño, además, se obtiene un coeficiente elevado que denota una fuerte relación entre las variables.

Analicemos ahora la relación con la otra variable categórica empleada para medir la diversificación geográfica.

\section{Tabla 11}

\section{PRUEBA U DE MANN-WHITNEY PARA TAMAÑO Y GRADO DE DIVERSIFICACIÓN GEOGRÁFICA PDI-GDI}

\begin{tabular}{|c|c|c|c|c|}
\hline & $\begin{array}{c}\text { Nivel de diversificación } \\
\text { geográfica }\end{array}$ & $\mathbf{N}$ & $\begin{array}{c}\text { Rango } \\
\text { Promedio } \\
\end{array}$ & Estadístico \\
\hline \multirow{3}{*}{ Tamaño } & PDI & 41 & 25,39 & \multirow{3}{*}{$\begin{array}{c}180,00 \\
(\text { sig. } 0,000)\end{array}$} \\
\hline & GDI & 20 & 42,50 & \\
\hline & Total & 61 & & \\
\hline \multirow{3}{*}{ Antigüedad } & PDI & 41 & 27,33 & \multirow{3}{*}{$\begin{array}{c}259,500 \\
(\operatorname{sig} .0,021)\end{array}$} \\
\hline & GDI & 20 & 38,53 & \\
\hline & Total & 61 & & \\
\hline
\end{tabular}

De nuevo, la relación con las variables tamaño y antigüedad resulta ser estadísticamente significativa. En este caso al $1 \%$ y al $5 \%$, respectivamente. De esta forma, un mayor tamaño y una mayor antigüedad van asociados con una mayor diversificación geográfica. Es decir, las empresas con Gran Diversificación Internacional son las que presentan un mayor tamaño y una antigüedad media mayor. 


\section{CONCLUSIONES}

El crecimiento internacional de las cadenas hoteleras españolas no se ha detenido a pesar de la crisis por la que ha atravesado la economía española durante los últimos años. Las ventajas que la diversificación geográfica tiene para las empresas, al reducir el riesgo o al permitir acceder a mercados con grandes oportunidades, pueden justificar dichas estrategias. En relación al estudio de dichas ventajas, uno de los temas que más interés ha suscitado ha sido la influencia de la diversificación internacional sobre los resultados empresariales. No obstante, en el sector hotelero español los estudios realizados sobre este tema son todavía escasos. Es por ello que nuestro trabajo se ha centrado en analizar la relación entre la diversificación geográfica y la rentabilidad empresarial de las cadenas hoteleras españolas con presencia internacional.

Los datos de nuestro estudio muestran una tendencia creciente hacia la internacionalización, al aumentar durante el período analizado el número de establecimientos y de plazas hoteleras en el exterior, así como el número medio de países distintos donde las empresas hoteleras españolas están presentes. Los datos también permiten observar que la principal zona hacia la que se han internacionalizado las empresas de nuestra muestra es la del Caribe, seguida de Europa y la Cuenca Mediterránea.

El análisis empírico realizado no permite confirmar que exista relación entre la diversificación geográfica y la rentabilidad económica que presentan las cadenas hoteleras españolas en el año 2014. Este resultado coincide con el obtenido por Lee (2008) para el caso de los hoteles de Estados Unidos, cuando analizó la relación lineal entre el valor de la empresa y el nivel de internacionalización. Sin embargo, sí observó que existía una relación curvilínea y significativa entre estas dos variables, al igual que Brida, RamónRodríguez, Such-Devesa y Driha (2016) para el caso de las empresas hoteleras españolas. En este último trabajo, los autores llegan a la conclusión de que existe un nivel de internacionalización más allá del cual los resultados de las empresas empiezan a disminuir. Por tanto, una posible explicación del resultado obtenido en nuestro trabajo sea la existencia de una relación curvilínea entre el grado de diversificación geográfica y la rentabilidad empresarial.

Por otra parte, tampoco hemos podido confirmar que exista una zona de internacionalización con una rentabilidad claramente superior a las otras. Únicamente se ha podido confirmar estadísticamente que las empresas cuya principal zona de internacionalización es el Caribe, tienen una rentabilidad mayor que las empresas cuya principal zona es la del resto de América. No obstante, la relación de la diversificación geográfica sí resultó ser positiva y significativa con otras variables empresariales analizadas como el tamaño y la antigüedad. En este sentido, se obtuvo que las empresas más diversificadas, desde el punto de vista geográfico, eran también las de mayor tamaño y las de mayor antigüedad.

Los resultados obtenidos pueden deberse a las limitaciones que presenta el trabajo, lo que nos abre al mismo tiempo futuras líneas de investigación y mejora. Como primera limitación, podemos señalar las medidas utilizadas. Las variables empleadas, tanto para medir la diversificación geográfica como la rentabilidad empresarial, podrían ser revisadas en investigaciones futuras. Por ejemplo, como aproximación al grado de internacionalización se podría utilizar el volumen de facturación de la empresa en el exterior sobre el 
volumen de facturación total. En cuanto a la rentabilidad, otros trabajos han empleado como medida la Rentabilidad sobre las Ventas (ROS), la Rentabilidad sobre la Inversión (ROI) o la denominada $q$ de Tobin. También se podría recurrir a otras medidas no financieras para analizar el éxito de las empresas, empleadas en trabajos que se han centrado en el sector hotelero, como propone Sainaghi (2010). Igualmente, la definición de las áreas geográficas podría ser diferente, distinguiendo un mayor número de zonas para poder estudiar más en detalle las diferencias entre ellas.

Otra limitación es el carácter secundario de las fuentes de datos consultadas. Como consecuencia, los datos manejados podrían no reflejar toda la realidad y podrían ser incompletos en caso de habernos dejado fuera alguna observación. En futuros trabajos se debería intentar acceder a información primaria de las empresas.

Finalmente, se debe tener en cuenta que los análisis realizados tienen un carácter limitado al ser bivariables. Sería necesario realizar un análisis multivariable donde se pudiera analizar la influencia conjunta de la diversificación geográfica y todo un conjunto de otras variables en los resultados empresariales. Entre ellas, la distancia cultural con los países donde la empresa tiene presencia internacional, las estrategias de entrada empleadas para establecerse en cada país, el tamaño y la antigüedad de la empresa, la diversificación de producto o la estructura de propiedad de los hoteles de la cadena. Todo ello nos abre futuras vías para seguir investigando sobre este tema.

\section{BIBLIOGRAFÍA}

AMIT, R. y LIVNAT, J. (1988): "Diversification strategies, business cycles and economic performance", Strategic Management Journal, vol. 9, pp. 99-110.

BENITO, D. (2009): “Análisis teórico de la relación entre diversificación corporativa y resultados empresariales”, Investigaciones Europeas de Dirección y Economía de la Empresa, vol. 15, n 2, pp. 105-126.

BERBEL, J.M. y RAMÍREZ, J.M. (2005): “La internacionalización del sector hotelero español: evolución y situación actual de las operaciones en el exterior", XV Jornadas Hispano-Lusas de Gestión Científica. Sevilla, 2-4 de febrero de 2005.

BERGER, P.G. y OFEK, E. (1995): “Diversification's effect on firm value", Journal of Financial Economics, vol. 37, $\mathrm{n}^{\mathrm{o}}$ 1, pp. 39-65.

BETTIS, R.A. (1981): "Performance differences in related and unrelated diversified firms", Strategic Management Journal, vol. 2, pp. 379-393.

BRIDA, J.G., DRIHA, O., RAMÓN-RODRÍGUEZ, A.B. y SCUDERI, R. (2015): “Dynamics of internationalisation of the hotel industry: the case of Spain", International Journal of Contemporary Hospitality Management, vol. 27, no 5 pp. 1024-1047.

BRIDA, J.G., RAMÓN-RODRÍGUEZ, A.B., SUCH-DEVESA, M.J. y DRIHA, O. (2016): "The inverted-U relationship between the degree of internationalization and performance: The case of Spanish hotel chains", Tourism Management Perspectives, vol. 17 , pp. 72-81.

CAMISÓN, C. (2000): "Strategic attitudes and information technologies in the hospitality business: an empirical analysis", International Journal of Hospitality Management, vol. 19, n 2 , pp. 125-143. 
CAPAR, N. y KOTABE, M. (2003): "The relationship between international diversification and performance in service firms", Journal of International Business Studies, $\mathrm{n}^{\circ}$ 34, pp. 345-355.

CHEN, C.-M. y CHANG, K.-L. (2012): "Diversification strategy and financial performance in the Taiwanese hotel industry", International Journal of Hospitality Management, $\mathrm{n}^{\mathrm{o}} 31$, pp. 1030-1032.

CHRISTENSEN, H.K. y MONTGOMERY, C.A. (1981): “Corporate Economic Performance: Diversification Strategy Versus Market Structure”, Strategic Management Journal, vol. 2, n 4, pp. 327-343.

CLAVER, E., ANDREU, R. y QUER, D. (2006): "Las ventajas de la diversificación estratégica para las empresas turísticas españolas. Una visión desde la Teoría de Recursos", Cuadernos de Turismo, $\mathrm{n}^{\circ} 17$, pp. 53-75.

CLAVER, E., ANDREU, R. y QUER, D. (2007): "La diversificación de las empresas hoteleras españolas: una explicación basada en la teoría de recursos", Revista de Investigaciones Europeas de Dirección y Economía de la Empresa, Vol. 13, nº 1, pp. 57-77.

CLAVER, E., QUER, D. y ANDREU, R. (2006): “La empresa hotelera española y sus decisiones de inversión directa en el exterior: influencia del país de destino y los factores empresariales", Revista Análisis Turístico, no 2, pp. 56-68.

COPLAN, A.M. (2008): “Are strategy-performance relationships contingent on macroeconomic environments?, Asia Pacific Journal of Management, vol. 25, pp. 635-665.

CORRAL, J.A. (2014): "La investigación turística en España”. En GARCÍA SÁNCHEZ, A. (coord.): 20 años de la actividad turística en España. Madrid, Síntesis, pp. 321-336.

DAVIS, P.S., ROBINSON, R.B. Jr., PEARCE II, J.A. y PARK, S.H. (1992): "Business unit relatedness and performance: a look at the pulp and paper industry", Strategic Management Journal, vol. 13, $\mathrm{n}^{\circ}$ 5, pp. 349-361.

DELIOS, A. y BEAMISH, P.W. (1999): “Geographic scope, product diversification and the corporate performance of Japonese firms", Strategic Management Journal, vol. 20, pp. 711-727.

DRIHA, O. y RAMÓN-RODRÍGUEZ, A. (2011): “Determinantes de la elección del modo de entrada de las hoteleras españolas en mercados foráneos". Papeles de Economía Española, ${ }^{\circ} 128$, pp. 296-315.

DUBOFSKY, P. y VARADARAJAN, P. (1987): "Diversification and measures of performance: additional empirical evidence", Academy of Management Journal, vol. 30, $\mathrm{n}^{\mathrm{o}}$ 3, pp. 597-608.

EXCELTUR (2015): Perspectivas turísticas. Valoración empresarial del segundo trimestre de 2015 y perspectivas para el trimestre de verano y el conjunto del año. $\mathrm{N}^{\circ} 53$, julio de 2015. Disponible en: http://www.exceltur.org/wp-content/uploads/2015/07/InformePerspectivas-N53-Balance-IIT-2015-y-perspectivas-para-el-verano-web.pdf.

FIGUEROLA, M. (2014): "Principales Magnitudes". En PARDELLAS DE BLAS, X. y MONDÉJAR JIMÉNEZ, J.A. (Directs.): La actividad turística española en 2013. Madrid, Síntesis, pp. 29-37.

GARCÍA, A. (2014): "Balance de dos décadas del turismo en España". En GARCÍA SÁNCHEZ, A. (coord.): 20 años de la actividad turística en España. Madrid, Síntesis, pp. 21-31. 
GERINGER, J.M., BEAMISH, P.W. y daCOSTA, R.C. (1989): "Diversification strategy and internationalization: implications for MNE performance", Strategic Management Journal, vol. 10, n 2, pp. 109-119.

GERINGER, J.M., TALLMAN, S. y OLSEN, D.M. (2000): "Product and geographic diversification among Japanese multinational firms", Strategic Management Journal, vol. 21, pp. 51-80.

GOMES, L. y RAMASWAMY, K. (1999): “An empirical examination of the form of the relationship between multinationality and performance", Journal of International Business Studies, vol. 30, n ${ }^{\circ}$, pp. 173-188.

GRANT, R.M., JAMMINE, A.P. y THOMAS, H. (1988): "Diversity, diversification, and profitability among British manufacturing companies, 1972-84”, Academy of Management Journal, vol. 31, $\mathrm{n}^{\circ}$ 4, pp. 771-801.

HALL, E.H. y JOHN, C.H. (1994): "A methodological note on diversity measurement", Strategic Management Journal, vol. 15, pp. 153-168.

HENNART, J.F. (2007): “The Theoretical Rationale for a Multinationality-Performance Relationship". Management International Review, vol. 47, $\mathrm{n}^{\circ}$ 3, pp. 423-452.

HITT, M.A., HOSKISSON, R.E. y KIM, H. (1997): "International diversification: effects on innovation and firm performance in product-diversified firms", Academy of Management Journal, vol. 40, $\mathrm{n}^{\circ}$ 4, pp. 767-798.

HOTELS (2015): “Hotels 325”. Hotels Magazine, July/August 2015. Disponible en: http:// www.hotelsmag.com.

HOSKISSON, R.E. y HITT, M.A. (1990): “Antecedents and performance outcomes of diversification: a review and critique of theoretical perspectives", Journal of Management, vol. 16, n 2, pp. 461-509.

HUERTA, P. y NAVAS, J.E. (2007): “Análisis de la relación entre la diversificación y los resultados empresariales: una revisión teórica”, Análisis Económico, n 49, vol. XXII, pp. 133-148.

INE (2015): Encuesta de Ocupación de Alojamientos Turísticos (Establecimientos hoteleros). Disponible en: http://www.ine.es/inebmenu/mnu_hosteleria.htm\#1.

INE (2013): Cuenta Satélite del Turismo de España. Base 2008. Serie 2008-2012. Disponible en: http://www.ine.es/prensa/np829.pdf.

JIMÉNEZ-PALMERO, A. y BENITO-OSORIO, D. (2011): "Geographical and product diversification in Spanish multinational companies", Esic Market, september-december 2011, pp. 647-676.

KIM, W.C., HWANG, P. y BURGERS, W.P. (1993): “Multinationals' diversification and the risk-return trade-off”, Strategic Management Journal, vol. 14, pp. 275-286.

KRANENBURG, van H., HAGEDOORN, J. y PENNINGS, J. (2003): "Measurement of International and Product Diversification in the Publishing Industry", European International Business Academy, 29th annual conference.

LEE, S. (2008): "Internationalization of US multinational hotel companies: Expansion to Asia versus Europe", International Journal of Hospitality Management, vol. 27, $\mathrm{n}^{\circ} 4$, pp. 657-664.

LEE, M.J. y JANG, S. (2007): "Market diversification and financial performance and stability: A study of hotel companies", Hospitality Management, vol. 26, pp. 362-375. 
LI, S.X. y GREENWOOD, R. (2004): "The effect of within-industry diversification on firm performance: sinergy creation, multi-market contact and market structuration", Strategic Management Journal, vol. 25, nº 12, pp. 1131-1153.

LÓPEZ, M.C. (2010): "Nuevo paradigma en turismo: un análisis desde la administración de empresas a partir de las publicaciones en revistas especializadas". En VALDÉS, L., DEL VALLE, E. y PULIDO, J.I. (Directs.): Retos para el turismo español. Cambio de paradigma, XIV Congreso AECIT, 18-20 noviembre de 2009, Gijón, pp. 91-102.

LU, J.W. y BEAMISH, P.W. (2001): "The internationalization and performance of SMEs", Strategic Management Journal, vol. 22, pp. 565-586.

LU, J.W. y BEAMISH, P.W. (2004): "International diversification and firm performance: The S-curve hypothesis", Academy of Management Journal, vol. 47, n 4, pp. 598-609.

LUBATKIN, M. y CHATTERJEE, S. (1994): "Extending modern portfolio theory into the domain of corporate diversification: does it apply?", Academy of Management Journal, vol. 37, nº 1, pp. 109-136.

MARKIDES, C.C. y WILLIAMSON, P.J. (1994): "Related diversification, core competences and corporate performance", Strategic Management Journal, vol. 15, pp. 149-165.

MARTORELL, O. y MULET, C. (2013): "Las empresas hoteleras". En PARDELLAS DE BLAS, X. Y MONDÉJAR JIMÉNEZ, J.A. (Directs.): La actividad turística española en 2013. Madrid, Síntesis, pp. 59-67.

MARTORELL, O., MULET, C. y OTERO, L. (2013): “Choice of market entry mode by Balearic hotel chains in the Caribbean and Gulf of Mexico". International Journal of Hospitality Management, vol. 32, pp. 217-227.

MONTGOMERY, C.A. y WERNERFELT, B. (1988): "Diversification, Ricardian rents, and Tobin's q", RAND Journal of Economics, vol. 19, $\mathrm{n}^{\circ}$ 4, pp. 623-632.

MORCK, R. y YEUNG, B. (1991): "Why investors value multinationality", Journal of Business, vol. 64, $\mathrm{n}^{\circ}$ 2, pp. 165-187.

PANDYA, A.M. y RAO, N.V. (1998): "Diversification and firm performance: an empirical evaluation", Journal of Financial and Satrategic Decisions, vol. 11, n 2, pp. 67-81.

PARK, C. (2002): "The effects of prior performance on choice between related and unrelated acquisitions: implications for the performance consequences of diversification strategy", Journal of Management Studies, vol. 39, n 7, pp. 1003-1019.

PARK, C. (2003): "Prior performance characteristics of related and unrelated acquirers", Strategic Management Journal, vol. 24, pp. 471-480.

PÉREZ, R. M. y CAZORLA, L. (2002): “Características del sector hotelero como factores determinantes de la estrategia de entrada en el exterior", Papers de Turisme, 31/32, 30-65.

PLA-BARBER, J., LEÓN-DARDER, F. y VILLAR, C. (2011): "The internationalization of soft-services: entry modes and main determinants in the Spanish hotel industry", Service Business, $\mathrm{n}^{\circ}$ 5, pp. 139-154.

RAMÍREZ, M. y ESPITIA, M.A. (2000): "El impacto de la estrategia de diversificación de producto e internacional sobre los resultados corporativos de las grandes empresas españolas", Cuadernos de Economía y Dirección de la Empresa, n 5, pp. 111-131.

RAMÍREZ, M. y ESPITIA, M.A. (2002): “The impact of product diversification strategy on the corporate performance of large Spanish firms", Spanish Economic Review, $\mathrm{n}^{\circ}$ 4, pp. 119-137. 
RUMELT R.P. (1974): Strategy, Structure and Economic Performance. Boston, Division of research Harvard Business School.

RUMELT, R.P. (1982): "Diversification Strategy and Profitability”, Strategic Management Journal, vol.3, pp. 359-369.

SAINAGHI, R. (2010): "Hotel performance: state of the art", International Journal of Contemporary Hospitality Management, vol. 22, n ${ }^{\circ}$ 7, pp. 920-952.

SINGH, A. y GU, Z. (1994): "Diversification, financial performance, and stability of foodservice firms", Hospitality Research Journal, vol. 18, n 2, pp. 3-18.

TALLMAN, S. y LI, J. (1996): "Effects of international diversity and product diversity on performance of multinational firms", Academy of Mangement Journal, vol. 39, $\mathrm{n}^{\circ}$ 1, pp. 179-196.

UNWTO (2015): Panorama OMT del turismo internacional. Edición de 2015. Disponible en: http://www.e-unwto.org/doi/pdf/10.18111/9789284416875.

VARADARAJAN, P. (1986): "Product Diversity and Firm Performance: An Empirical Investigation", Journal of Marketing, vol. 50, pp. 43-57.

VARADARAJAN, P. y RAMANUJAM, V. (1987): "Diversification and performance: a reexamination using a new two-dimensional conceptualization of diversity in firm", Academy of Management Journal, vol. 30, n 2, pp. 380-393. 
